\title{
Aleación mecánica: Método de obtención de polvos metálicos y de materiales compuestos ${ }^{(\cdot)}$
}

\author{
Elisa María Ruiz-Navas", Cesar Edil da Costa*, Francisco Velasco López" y \\ Jose Manuel Torralba Castelló*
}

\begin{abstract}
Resumen Este trabajo está dedicado al proceso de aleación mecánica como método de obtención de polvos. En él se repasan los aspectos más importantes implicados en el proceso, los fundamentos, así como su aplicación particular a la obtención de polvos metálicos.
\end{abstract}

Palabras clave Aleación mecánica. Materiales compuestos de matriz metálica. Endurecimiento por dispersión de óxidos.

\section{Mechanical alloying: A method to obtain metallic powders and composite materials}

\begin{abstract}
This work deals with the mechanical alloying process as a method for powder production. The main aspects involved are as well as the fundaments and their particular application to the metal powder production.

Keywords

Mechanical alloying. Metal matrix composites. Oxide dispersion strengthening.
\end{abstract}

\section{INTRODUCCIÓN}

Si bien el principal método comercial de fabricación de polvos metálicos aleados es la atomización, en los últimos años y coincidiendo con el desarrollo de los materiales compuestos de matriz metálica (MMC), están surgiendo procesos basados en la molienda como un método eficaz para conseguir, tanto aleaciones de difícil obtención por otros métodos como polvos de material compuesto. De todos ellos, uno de los más efectivos y de mayor interés es la aleación mecánica.

La atomización ha demostrado ser la técnica más favorable para la obtención de polvos prealeados, debido a la posibilidad de un buen control de la composición química, tamaño, forma y distribución granulométrica de los mismos. Estas características, importantes para la posterior compactación, añadidas a la posibilidad de obtener una microestructura fina con un elevado contenido en solutos, han hecho de la atomización el proceso de obtención de polvo de mayor implantación industrial.

- Frente a esto, la aleación mecánica constituye el proceso mecánico más actual para la obtención de polvo, al permitir obtener composiciones y niveles de propiedades imposibles de alcanzar por otros métodos, incluida la atomización.

Esta técnica fue estudiada y desarrollada por primera vez a mediados de los años 60 , por John $S$. Benjamin, en el laboratorio de investigación de INCO, en un esfuerzo por encontrar el modo de combinar el endurecimiento por dispersión de óxidos con el endurecimiento por precipitación con la fase $\gamma^{\prime}$ en superaleaciones base níquel para aplicaciones en turbinas de gas ${ }^{[1-5]}$. Desde entonces, la aleación mecánica ha diversificado su campo de aplicación desde las aleaciones base hierro a las aleaciones de aluminio y sistemas cerámicos, y está ya comercializando numerosos materiales.

Definido como un proceso de molienda en seco, la ventaja fundamental que presenta es, precisamente, la posibilidad de obtener composiciones inalcanzables por procesos como la atomización, elevando el contenido en solutos muy por encima de las composiciones de equilibrio y aumentando el porcentaje en refuerzos, eliminando los problemas de segregación y formación de aglomerados generalmente asociados a las mezclas pulvimetalúrgicas convencionales $\left.{ }^{[6 \text { y }} 7\right]$.

(•) Trabajo recibido el día 23 de septiembre de 1999 y aceptado en su forma final el 22 de mayo de 2000.

${ }^{*}$ Universidad Carlos III de Madrid. Dpto. Materiales. Avda. Universidad 30.28911 Leganés (Madrid, España).

$\left({ }^{* *}\right)$ Universidade do Estado de Santa Catarina. Dept. Ing. Mecánica. Campus Universitario Bom Retiro, 89223-100 Joinville /SC (Brasil). 


\section{PROCESO DE ALEACIÓN MECÁNICA}

La mezcla de polvo, introducida en el molino de bolas, es sometida a colisiones de alta energía por

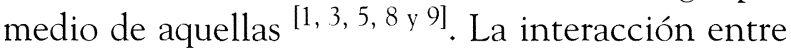
las bolas moledoras y las partículas de polvo puede caracterizarse por procesos como la soldadura en frío o la deformación plástica y posterior fragmentación de las partículas, hasta que se produce un polvo en el que cada partícula está constituida por la composición porcentual de la mezcla de polvo inicial ${ }^{[1,10 \text { y } 11]}$. Así, las continuas y repetidas soldaduras y fracturas son los más importantes procesos implicados que conducen a la transformación material de la estructura cristalina por reacciones en estado sólido ${ }^{[3,5 \text { y 8]. }}$.

La aleación mecánica presenta por ello, frente a otros métodos de obtención de polvo, la característica fundamental de realizarse en estado sólido, eliminando de esta forma las limitaciones asociadas a los puntos de fusión y solubilidades relativas. Permite así, la síntesis de nuevas aleaciones partiendo de una mezcla inicial de elementos de alta y baja temperatura de fusión, y la síntesis de aleaciones o materiales compuestos con componentes altamente dispersados, muy lejos de su estado de equilibrio térmico ${ }^{[12-24]}$

\subsection{Principales variables del proceso}

\subsubsection{Materias primas}

Es evidente que van a ser las características del polvo inicial las que determinen la evolución específica del proceso. Habitualmente, se parte de materiales en forma de polvos elementales o compuestos como polvos prealeados, carburos, óxidos, intermetálicos, etc.

Pero es, quizás, la respuesta específica del polvo a la hora de absorber la energía suministrada por las bolas lo que va a determinar el predominio de la soldadura o la fractura en el proceso, por lo que las mezclas pueden clasificarse según la ductilidad de la materia prima como ${ }^{[25]}$ :

a) dúctil-dúctil: en este caso hay un predominio de la soldadura frente a la fractura. Se forma una estructura laminar en las partículas aglomeradas y las láminas van desapareciendo con el tiempo de molienda, permitiendo obtener un material muy homogéneo. Se produce en el caso de la mezcla de dos materiales metálicos para obtener una aleación o intermetálico ${ }^{[25]}$. b) dúctil-frágil: en este caso se produce un proceso rápido de dispersión y posterior difusión, donde las partículas frágiles, según van fracturándose van introduciéndose dentro del material dúctil. Esta difusión se ve favorecida por el calentamiento inherente a la técnica. Al final, la homogeneización del producto puede ser parcial o total, dependiendo de la dispersión y, en su caso, de la solubilidad del elemento frágil en la matriz del elemento dúctil ${ }^{[26]}$.

c) frágil-frágil: en esta combinación se produce un claro predominio de la fractura. No obstante, el proceso de aleación da lugar a la formación de capas ultrafinas que pueden inducir a la formación de aleaciones ${ }^{[27]}$.

\subsubsection{Agente controlador del proceso}

A pesar de esta clasificación genérica, el proceso de aleación mecánica suele incluir, al menos, un componente dúctil que actúa como soporte o matriz de todos los demás componentes ${ }^{[5,9}$ y 28$]$. Es la tendencia específica de este a la soldadura la que exige la adición del agente controlador del proceso (PCA, Process Control Agent) ${ }^{[1,2 \text { y 10] }}$.

Las partículas de polvo, sometidas a colisiones de alta energía dentro del molino, evolucionan a través de continuas soldaduras y fracturas. Sólo un adecuado equilibrio entre ambos fenómenos permite el avance del proceso de forma adecuada. Para evitar la excesiva tendencia a la soldadura que presentan algunos materiales como el aluminio es necesaria la adición de dicho PCA. De otra forma, desemboca en la soldadura en frío de las partículas en las paredes del molino y en las bolas, imposibilitando el proceso.

Los PCA generalmente empleados se encuentran en forma de polvo, siendo absorbidos por las superficies de las partículas, con lo que se disminuye la tensión superficial y se evita la formación de aglomerados, permitiendo el avance del proceso ${ }^{[1,28}$ y 29$]$.

Un aspecto importante, asociado a la adición del PCA, lo constituye la contaminación derivada de su descomposición durante el proceso, en particular en materiales de elevada pureza ${ }^{[6}$ 30]. Dado que la mayoría de los PCA son compuestos orgánicos, cuya composición es, por tanto, carbono, oxígeno e hidrógeno, la contaminación en estos elementos resulta inevitable ${ }^{[31]}$. No obstante, no en todos los casos esta contaminación actúa en detrimento de las propiedades de los materiales. Existen materiales, como el aluminio, sus aleaciones y materiales compuestos, donde la incorporación del PCA da como resultado la dispersión de finas partículas de $\mathrm{Al}_{4} \mathrm{C}_{3}$ (Fig. 1). 


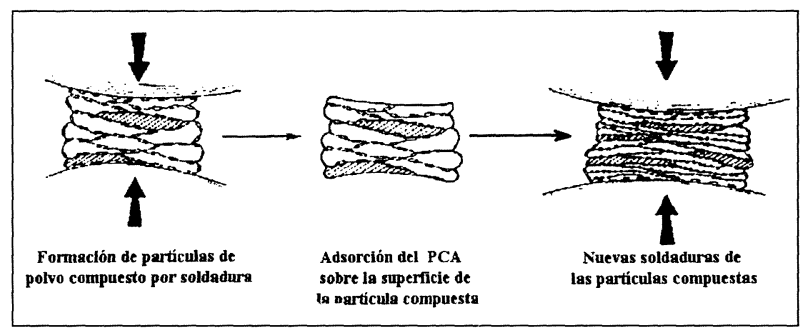

Figura 1. Esquema de la adsorción del PCA durante el proceso de aleación mecánica ${ }^{[39]}$.

Figure 1. Scheme of PCA adsortion during mechanical alloying process ${ }^{[39]}$.

Estas partículas, junto con las de $\mathrm{Al}_{2} \mathrm{O}_{3}$ procedentes de la superficie del polvo, provocan un endurecimiento por dispersión y, consecuentemente, un aumento en la dureza del polvo que repercute en las propiedades del material consolidado. Este endurecimiento es mayor al tratarse de una dispersión producida, en estas aleaciones, por aleación mecánica y por ello mucho más fina y homogénea que la alcanzada por otros métodos ${ }^{[32-41]}$.

\subsubsection{Relación bolas/carga}

La relación bolas/carga, indicada siempre en masa, es uno de los parámetros más importantes en la aleación mecánica. Su importancia radica en que el número de colisiones por unidad de tiempo crece a medida que lo hace el número de bolas ${ }^{[42}$ y 43$]$. De esta forma, a medida que se incrementa el número de colisiones se favorecen los fenómenos de soldadura en frío y fractura de las partículas de polvo atrapadas entre las bolas (Fig. 2), fenómenos inherentes al proceso, al mismo tiempo que aumenta localmente la temperatura de molienda, lo que provoca finalmente una aceleración en el proceso de difusión ${ }^{[44]}$. La selección óptima de la relación bolas/carga, que oscila generalmente entre 10:1 y 20:1 ${ }^{[28 \text { y } 45]}$ para un molino de alta energía depende, además, de otros parámetros y condiciones del proceso, como la velocidad, pero es principalmente el tipo de molino y su capacidad específica de suministrar energía al sistema lo que condiciona su elección. Existen estudios que confirman que, a medida que se incrementa la relación se produce un crecimiento en la dureza del polvo, al incrementarse la deformación plástica de las partículas de polvo; sin embargo, dicha relación no suele superar los rangos anteriormente indicados.

\subsubsection{Intensidad de molienda}

La intensidad de molienda constituye un parámetro crítico en la medida de la energía de molien-

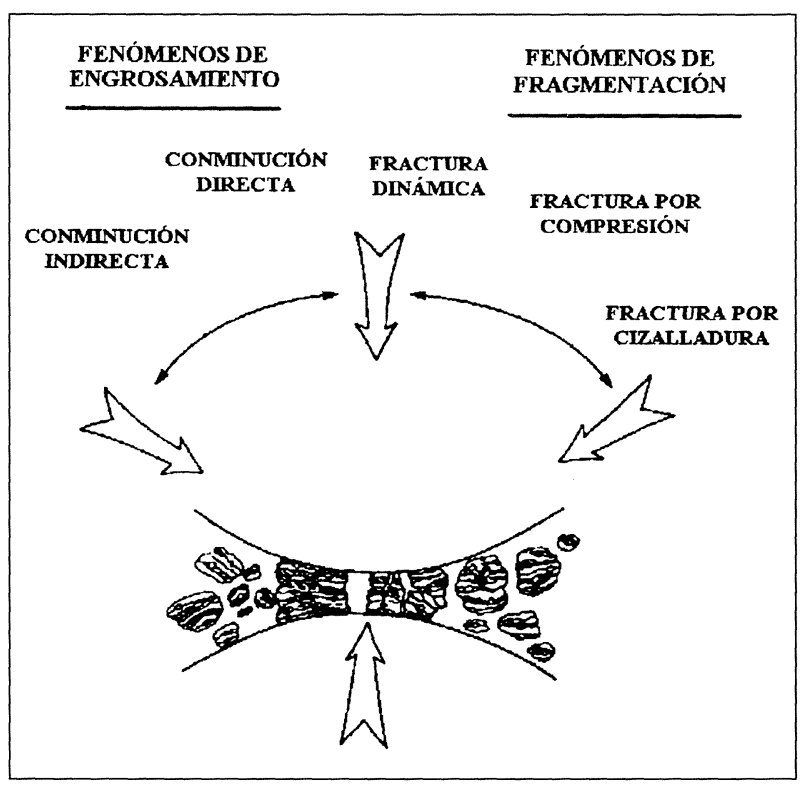

Figura 2. Esquema de la soldadura y fractura del polvo por acción de las bolas colisionando ${ }^{[42]}$.

Figure 2. Scheme of powder welding and fracture by balls colliding $^{[42]}$.

$\mathrm{da}^{[9}{ }^{9}{ }^{28]}$, la cual es directamente proporcional a las características del polvo y a las propiedades del mismo generadas durante el proceso. Es evidente que, cuando dicha intensidad se ve incrementada, bien por la cantidad de bolas bien por la densidad del material de las mismas o por el incremento de la velocidad de colisión, la energía transferida a las partículas es mucho mayor por cada colisión y, por tanto, mayor es la energía a disipar en forma de ca-

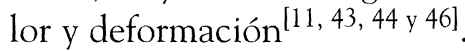

\subsubsection{Equipo de molienda}

Se considera equipo de molienda al sistema formado por la vasija, contenedor o molino y los cuerpos moledores. Son numerosos los tipos de molinos disponibles actualmente para la realización del proceso: molinos horizontales convencionales, molinos controlados por fuerzas magnéticas, molinos de impacto o excéntricos, destacando entre todos los molinos planetarios y los molinos de alta energía o attritor ${ }^{[42,45}$ y 47$]$.

Las diferencias de funcionamiento entre todos ellos, así como las velocidades disponibles están directamente relacionadas con su eficacia relativa así como con la energía de molienda suministrada por cada uno de ellos ${ }^{[8]}$.

No obstante, el diseño y el empleo de los molinos de alta energía (attritors) es lo que ha permitido el avance real del proceso de aleación mecánica. Estos molinos se caracterizan por contener unas hélices en 
su interior que son las que proporcionan el movimiento y la velocidad a las bolas, confiriendo al proceso una elevada energía de molienda ${ }^{[41,48 \text { y } 49]}$. Además, frente a otros, estos equipos permiten el empleo de atmósferas controladas que preserven el polvo de contaminaciones durante el proceso.

\subsubsection{Atmósfera controlada}

La atmósfera en la cual se lleva a cabo la molienda ejerce una gran influencia sobre la contaminación del polvo ${ }^{[21 \text { y 44] }}$. Para prevenir la oxidación y contaminación durante la aleación mecánica, el proceso generalmente se lleva a cabo en un molino que, previamente, ha sido desgasificado, y que está bajo presión y/o flujo de gas inerte.

En la mayoría de los casos, la atmósfera empleada es argón, aunque algunos autores emplean

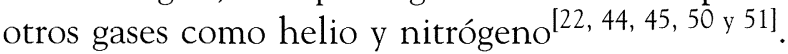
Estos, sin embargo, no suelen ser comunes, en particular el nitrógeno dada su reactividad y su tendencia a formar compuestos con el material de partida. Además, como se ha indicado anteriormente, no todos los equipos permiten llevar a cabo moliendas con flujo de atmósferas controladas.

No obstante, cada material requiere unas condiciones de proceso específicas ya que, en numerosas ocasiones, niveles de contaminación que son críticos a la hora de obtener un material no lo son en otros. Así ocurre con la obtención por aleación mecánica de materiales como los SAP (Sintered Aluminum Products) o DISPAL (Dispersion Strengthened Aluminum), donde la presencia de oxígeno procedente de la atmósfera repercute en una fina dispersión de $\mathrm{Al}_{2} \mathrm{O}_{3}$ dando lugar a aleaciones de aluminio, endurecidas por dispersión, de elevadas propiedades.

\subsection{Evolución y etapas principales}

Son numerosos los fenómenos que intervienen en el proceso de aleación mecánica, siendo igualmente complejo aislar las etapas a través de las cuales evoluciona el polvo hasta la consecución del elevado nivel de propiedades que los caracteriza.

Por ello, las etapas han sido descritas en numerosas ocasiones, pero casi siempre (Fig. 3), de forma cualitativa ${ }^{[2,8,10,42,46 \text { y 52] }}$. Describir dicha evolución, en función del tiempo y condiciones del proceso, depende directamente de las características iniciales del polvo que condicionan de forma determinante su evolución. Esta evolución viene determinada, en los materiales dúctiles, a través de

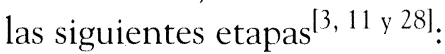

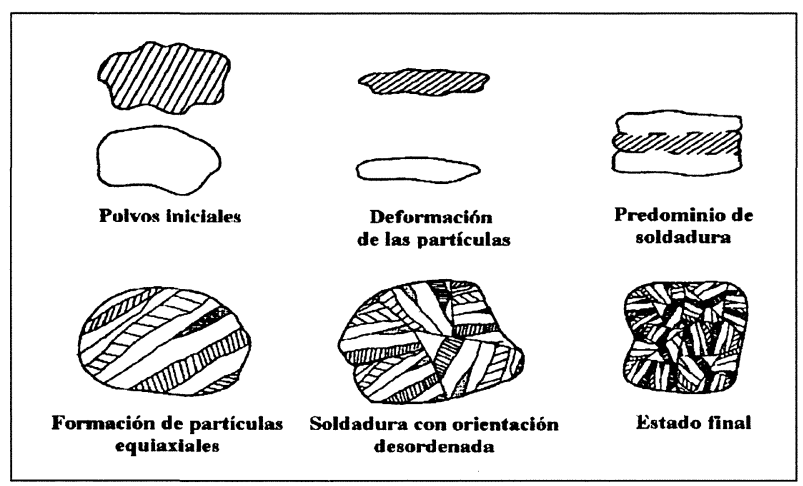

Figura $3^{[11]}$. Etapas de aleación mecánica en un material dúctil-dúctil. 1) Materia prima. 2) Inicial. Deformación de las partículas. 3) Predominio de soldadura. 4) Formación de partículas equiaxiales. 5) Soldadura con orientación marcadamente desordenada. 6) Final o afino de estructura, régimen permanente.

Figure $3^{[11]}$. Mechanical alloying steps for a ductile-ductile material. 1) Raw material. 2) Initial. Powder deformation. 3) Welding predominance. 4) Equiaxed particle formation. 5) Random welding orientation. 6) Steady state.

\subsubsection{Inicial}

Se produce en los primeros instantes de molienda y se caracteriza por la deformación del polvo que, atrapado entre las bolas, comienza a adquirir una morfología aplastada o en forma de láminas. Las partículas de polvo dúctiles son fácilmente deformadas ante la fuerza de compresión de las bolas que chocan entre sí, mientras que las partículas más duras ofrecen más resistencia a la deformación, fracturándose en algunos casos.

\subsubsection{Predominio de soldadura (welding predo- minance)}

Esta etapa se caracteriza por la formación de partículas con estructura sándwich o multicapa donde las láminas van soldándose paralelas entre sí por acción de los cuerpos moledores, incrementando de esta forma el espesor de las partículas. A pesar de ello, la morfología de las partículas es bastante aplastada, por lo que su volumen equivalente es muy superior al que realmente poseen.

\subsubsection{Formación de partículas equiaxiales (equia- xed particle formation)}

El proceso evoluciona a través de la formación de partículas equiaxiales, probablemente debido a la pérdida de ductilidad del material, que endureciéndose a lo largo de las diferentes etapas presenta una mayor tendencia a la conminucion. 


\subsubsection{Soldadura con orientación marcadamente desordenada (random welding orientation)}

En esta etapa se produce un fuerte incremento en la fracción de partículas que, soldándose sin ningún tipo de preferencia en la orientación, adquieren una morfología ya definitivamente equiaxial.

Esta evolución va asociada con un progresivo incremento de propiedades del polvo, manifestadas principalmente a través de la dureza.

\subsubsection{Final o afino de estructura, régimen perma- nente (steady state)}

El proceso avanza finalmente a través del crecimiento en la homogeneidad interna del polvo y el afino de su microestructura. No aparecen ya estructuras en forma de capas, ya que estas llegan a ser tan finas $(<0,5 \mu \mathrm{m})$ que dejan de ser visibles, alcanzándose de esta forma en cada partícula de polvo la composición deseada. Se constituye un polvo prealeado equivalente a la composición porcentual de la mezcla de polvo inicial.

El establecimiento del régimen permanente pasa también por un equilibrio en los procesos de fractura y soldadura, evolucionando finalmente a través del afino microestructural y crecimiento en la microdureza del polvo que, evidentemente, se manifiesta en una disminución de su ductilidad y, por consecuencia, en una progresiva disminución del tamaño.

La evolución de la estructura en los materiales constituidos por mezclas dúctil-frágil, como son los materiales compuestos de matriz metálica, es algo diferente a la anteriormente descrita. En estos, el metal o la aleación que compone la matriz va constituyéndose a partir de los polvos elementales, al mismo tiempo que actúa como soporte del refuerzo, que, mucho más duro, va fracturándose y distribuyéndose homogéneamente en la matriz hasta quedar completamente embebido en ella (Fig. 4).

\section{Materiales obtenidos por aleación mecánica}

Desde la introducción del proceso y la obtención industrial por aleación mecánica de aleaciones base níquel endurecidas por dispersión de óxidos en los años 60 , se ha desarrollado un gran número de aplicaciones del proceso a la obtención de otros materiales. En particular, son numerosas las aleaciones, intermetálicos, materiales amorfos y materiales compuestos desarrollados ${ }^{[24] .}$

El desarrollo de este proceso ha llegado a la obtención de materiales convencionales como las

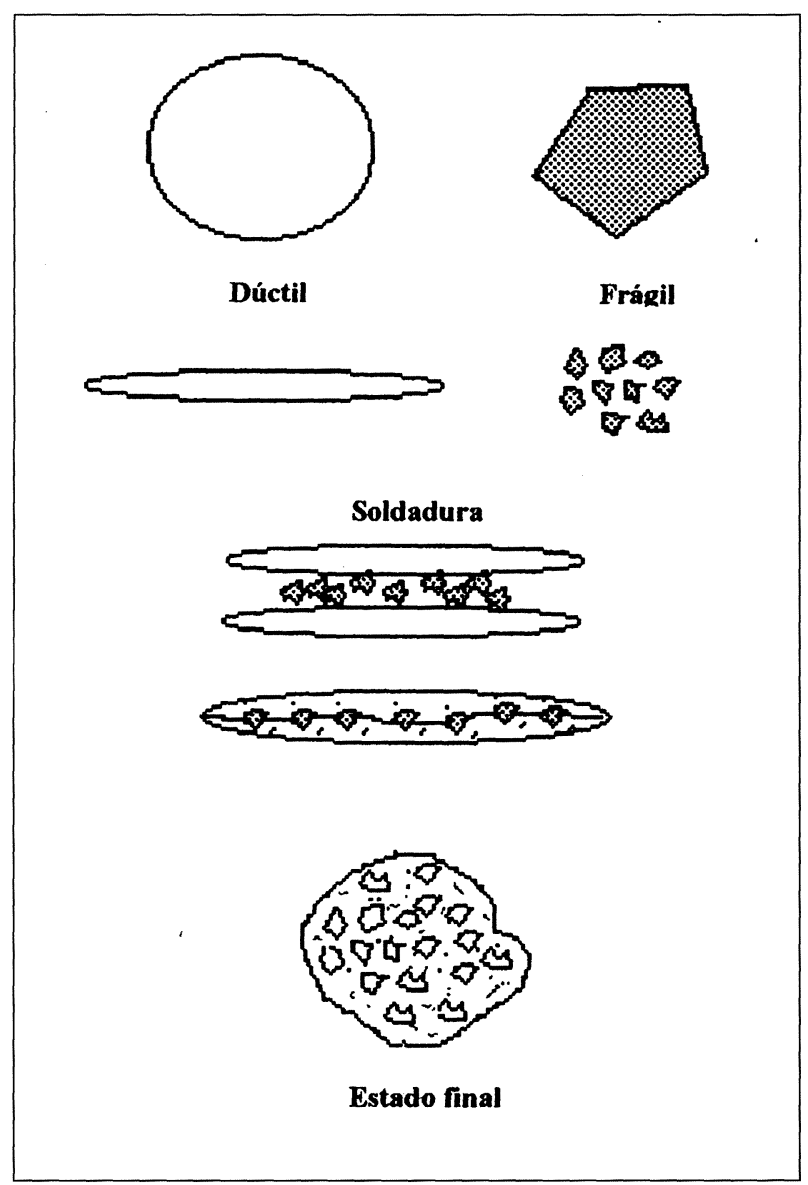

Figura 4. Etapas de aleación mecánica en un material dúctil-frágil. 1) Materia prima. 2) Inicial. Deformación y fractura de las partículas. 3) Predominio de soldadura, formación del polvo compuesto. 4) Final o afino de estructura, régimen permanente.

Figure 4. Mechanical alloying steps for a ductile-brittle material. 1) Raw material. 2) Initial. Deformation and fracture of the particles. 3) Welding predominance, composite powder formation. 4) Steady state.

aleaciones base hierro ${ }^{[4}$ y 53] o los aceros ferríticos endurecidos por dispersión, con un buen nivel de propiedades y resistencias a la oxidación a temperaturas elevadas superiores a las obtenidas por metalurgia convencional ${ }^{[4}$ y 21$]$.

De igual forma, el fuerte crecimiento en las propiedades mecánicas del cobre y sus aleaciones, derivadas del endurecimiento por dispersión, sin detrimento de la conductividad eléctrica, ha permitido, gracias a la aleación mecánica, ampliar en gran medida el campo de aplicación de estas aleaciones ${ }^{[4 \text { y } 21]}$.

La posibilidad de extensión de los límites de solubilidad, restringida en otros métodos de obtención de polvo, incluso en la solidificación rápida, ha hecho posible el desarrollo, no sólo de las aleaciones de titanio con elevados contenidos en tierras raras como erbio, itrio, gadolinio y neodimio, sino también la obtención de fases amorfas e 
intermetálicos base titanio como $\mathrm{TiAl}_{\text {y }} \mathrm{Ti}_{3} \mathrm{Al}$. Junto con ellas, actualmente se emplea la aleación mecánica para obtener materiales con una elevada

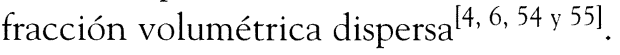

El proceso de aleación mecánica también está potenciando la obtención de un amplio espectro de materiales, desde las aleaciones base cobalto, expuestas a condiciones de fuerte desgaste, las de base magnesio, permitiendo un exacto control de la composición y microestructura, hasta las aleaciones base niobio ${ }^{[56]}$.

Especial interés tiene la obtención de compuestos intermetálicos, de aplicación creciente en estos días, por la excelente combinación de propiedades que ofrecen. Sin embargo, las dificultades presentes en su obtención, en numerosas ocasiones asociadas a procesos fuertemente exotérmicos, hacen necesario un gran control de su proceso de síntesis, lo que queda garantizado mediante su obtención

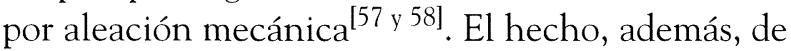
que el proceso se realice completamente en estado sólido elimina las limitaciones de inmiscibilidad y solubilidad propios de otros procesos. En la actualidad, se está desarrollando un amplio rango de composiciones desde las indicadas de base titanio a las de base aluminio como $\mathrm{Al}_{3} \mathrm{Ti}^{[59]}$, las de base níquel, donde destacan el $\mathrm{NiAl}$ y $\mathrm{Ni}_{3} \mathrm{Al}^{[25,60 \text { y 61] }} \mathrm{y}$ las de base hierro ${ }^{[24}$ y 25$]$.

De igual forma, aunque con menos profusión, se está empleando el proceso de aleación mecánica

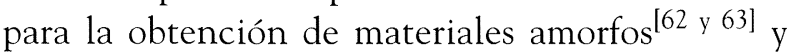
nanocristalinos, generalmente compuestos por metales de transición como el zirconio, el hafnio o el lantano, mostrando una gran versatilidad al eliminar las restricciones impuestas por los métodos de aleación convencionales.

Una especial atención merecen las aleaciones de aluminio pertenecientes a las distintas series, así como los materiales compuestos obtenidos a partir de estas. La primera aplicación de este proceso a los materiales de matriz aluminio fue la obtención de aluminio sin alear, endurecido por dispersión, principalmente de partículas de $\mathrm{Al}_{2} \mathrm{O}_{3}$ y $\mathrm{Al}_{4} \mathrm{C}_{3}$, mostrando resultados prometedores frente al metal sin reforzar. Posteriormente, el empleo de esta técnica para el aluminio y sus aleaciones, así como para la obtención de aleaciones con dispersión de óxidos (ODS), ha crecido de forma vertiginosa, dando como resultado una amplia gama de aleaciones y productos, como los ya indicados SAP y DISPAL, que permiten elevar el rango de temperaturas de trabajo en servicio de las aleaciones base aluminio.
A partir de éstas, han sido numerosas las composiciones base aluminio desarrolladas, dada la versatilidad y el control exacto de la composición que la aleación mecánica permite, destacando entre todas ellas las $\mathrm{Al}-\mathrm{Cu}-\mathrm{Mg}^{[64}$ y ${ }^{65]}$, las $\mathrm{Al}-\mathrm{Mg}-\mathrm{X}^{[34,40,65 \text { y } 66]}$ y las Al-Fe- $\mathrm{X}^{[4,17,22,66 \text { y } 67] .}$

Otra de las aplicaciones importantes de este proceso es la posibilidad de obtención de materiales compuestos de matriz metálica. La gran ventaja que la aleación mecánica ofrece estriba en la posibilidad de obtener propiedades muy superiores a las alcanzadas por otros métodos, ya que elimina uno de los mayores problemas asociados a su producción como es la formación de segregaciones y aglomerados. La posibilidad de obtener un material compuesto constituido, ya desde el principio, por polvos de matriz metálica reforzados por partículas cerámicas, finamente dispersas en su interior $^{[50,68 \text { y } 69]}$, permite alcanzar un material final de propiedades muy por encima de las esperadas en este tipo de materiales ${ }^{[52 \text { y } 70-72]}$.

El amplio desarrollo de todas estas aleaciones se debe, principalmente, a los buenos resultados alcanzados en todas ellas, superando las características y temperaturas de aplicación de los materiales obtenidos por metalurgia convencional y solidificación rápida. Estos resultados se deben, tal y como se ha indicado anteriormente, a la disminución alcanzada en el tamaño de grano (entre 0,2 y $0,5 \mu \mathrm{m})^{[41 \text { y } 73]}$, a la elevada densidad de dislocaciones así como a la dispersión de óxidos inherentes

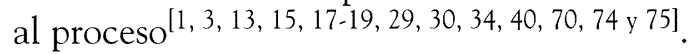

En los últimos años se están desarrollando numerosas investigaciones en el campo de aplicación de este proceso, abriendo progresivamente el abanico de sus posibilidades. La posibilidad de obtener materiales ya convencionales con propiedades muy superiores, así como superar las limitaciones presentes en métodos de fabricación perfectamente establecidos, permite atisbar un futuro prometedor a medio y largo plazo para esta técnica.

\section{REFERENCIAS}

[1] J.S. Benjamin, Mod. Dev. Powder Metall. 21 (1988) 397-414.

[2] J.S. Benjamin, Metal Powder Rep. 44 (1990) 122-127.

[3] J.S. Benjamin y T.E. Violin, Metall. Trans. 5 (1974) 1929-1934.

[4] I.S. Polking y A.B. Borzov, Adv. Perf. Mater. 2 (1995) 99-109.

[5] J.S. Benjamin, Adv. Powder Metall. Particulate Mater. 7 (1992) 155-168. 
[6] R.W. Gardiner, P.S. GoOdWIN, S.B. DOdd y B.W. VineY, Adv. Perf. Mater. 3 (1996) 343-364.

[7] F.H. Froes, C. Suryanarayana, P.R. Taylor, C.M. WardClose y P. GoOdWIN, Powder Metal. 39 (1996) 63-39.

[8] J.S. BenJamin, Metall. Trans. 1 (1970) 2943-2951.

[9] L. LÜ, M.O. Lal, S. Zhang, J. Mater. Proc. Tech. 67 (1997) 100-104.

[10] S. Benjamin y R.D. Schelleng, Metall. Trans. A. 12 (1981) 1827-1832.

[11] B.J.M. AIKIN y T.H. COURTNeY, Metall. Trans. A. 24 (1993) 647-657.

[12] P.K. Mirchandani, D.O. Gothard, A.I. KempPinen, Adv. Powder Metal. 3 (1989) 161-173.

[13] K.M. LeE, J.H. LeE e I.H. Moon, Proc. PM'93, Kyoto, Japón, 1993, Japan Society of Powder Metallurgy, Kyoto, Japón, 1993, pp.116-119.

[14] I.H. MOON, J.H. LeE, K.M. LEE Y Y.D. KIM, Proc. PM'94, vol.III, París, Francia, 1994, SF2M, París, Francia, 1994, pp. 1643-1646.

[15] A. Layyous, S. Nadiv e I.J. LIN, Powder Metall. Int. 22 (1990) 21-24.

[16] L. Froyen, L. Delaey, X.P. Niu, P. Le Brun y C.Peytour, JOM. 45 (1995) 16-19.

[17] N. Xiaoping, P. le Brun, L. Froyen, C. Peytour y L. Delaey, Adv. Powder Metall. Particulate Mater. 7 (1992) 271-283.

[18] R.S. Mishra, T.R. Bieler y A.K. MukherJee, Scr. Metall. Mater. 26 (1992) 1605-1608.

[19] K. HIGASHI, Proc. PM'93, Kioto, Japón, 1993, Japan Society of Powder Metallurgy, Kioto, Japón, 1993, pp. 587-590.

[20] J.A. HAWK, J.K. BRIGGS y H.G.F. WILSDORF, Adv. Powder Metall. 3 (1989) 285-299.

[21] F.H. Froes y C. Suryanarayana, Metal Powder Rep. 14 (1994) 14-18.

[22] F. Cardellini, V. Contini, y G. Mazzone, J. Mater. Sci. 31 (1996) 4175-4180.

[23] X. NIU y L. LU, Adv. Perf. Mater. 4 (1997) 275-283.

[24] S. Van Dyck, L. Delaey, L. Froyen y L. Buekenhout, Acta Mater. 46 (1998) 2831-2840.

[25] C.E. DA COSTA, Tesis Doctoral, ETSI de Minas de Madrid, Universidad Politécnica de Madrid, 1998.

[26] E.M. RuIz-NaVAS, Tesis Doctoral, ETSI de Minas de Madrid, Universidad Politécnica de Madrid, 1999.

[27] R.M. DAVIS y C.C. KoCH, Scr. Metall. 21 (1987) 305310.

[28] L. Lü y M.O. LAl, Mechanical Alloying, Ed. Kluwer Academic Publishers, Amsterdam, Holanda, 1998 pp. 1-64.

[29] Y.C. Cheng, S.H. Wang, P.W. KaO y C.P. Chang, Mater. Sci. Forum. 217-222 (1996) 1891-1896.

[30] R.D. SChelleng y S.J. DonaChie, Metal Powder Rep. 38 (1983) 575-576.
[31] M. HuLl, Powder Metall. 40 (1997) 106-109.

[32] T. Takahash y M. Motoyama, Adv. Powder Metall. Particulate Mater. 7 (1992) 413-420.

[33] J.S. Benjamin y M.J. BOMfORD, Metall. Trans. A. 8 (1977) 1301-1305.

[34] J.Kaneko, M. Sugamata, D.G. Kim y H. Higuch, Mater. Sci. Forum. 217-222 (1996) 919-924.

[35] H. Kreye, G. Reiners, M. Schubert, G.E. LÖHmer, Horizons Powder Metall. (1986) 707-711.

[36] X.P. Niu, L. Froyen, L. Delaey y C. Peytour, Proc. PM'94, vol.III, Paris, Francia, 1994, SF2M, París, Francia, 1994, pp.1617-1624.

[37] G. JANGG, M. Slesar, M. BesterCl, J. DuRISIN y K. SCHRÖDER, Powder Metall. Int. 21 (1989) 25-29.

[38] P. Le Brun, L. Froyen, B. Munar y L. Delaey, Proc. PM'90 World Conference on Powder Metallurgy, vol.2, Londres, Reino Unido, 1990, Institute of Materials, Londres, Reino Unido, 1990, pp.1-6.

[39] J. DurišIn, M. Orolínová y K. DurišInová, Adv. Powder Metall. Particulate Mater. 9 (1992) 195-208.

[40] R.D. SChelleng, P.S. GILman, A.D. JatKat y S.J. DONACHIE, Powder Metall. Defense. 6 (1984) 165-174.

[41] J.H. Weber y R.D. ShelleNG, Dispersion Strengthening of Aluminum alloys, Ed. Y-W.Kim y W.M. Griffith, TMS, 1988, pp. 467-481.

[42] D.R. Maurice y T.H. Courtney, Metall. Trans. A. 21 (1990) 289-303.

[43] D.R. Maurice y T.H. Courtney, Metall. Trans. A. 26 (1995) 2431-2435.

[44] G.B. SChaffer y P.G. MCCORMick, Metall. Trans. A. 23 (1992) 1285-1290.

[45] H. Zoz, D. ERnst, H. Weiss, M. Magini, C. Powell, C. Suryanarayana y F.H. Froes, Metall 50 (1996) 575579.

[46] D.R. Maurice y T.H. Courtney, Metall. Trans. A. 26 (1995) 2437-2444.

[47] M.L. Öveçoglu y B. ÖzKal, J. Mater. Res. 11 (1996) 1673-1682.

[48] H. Zoz, Metal Powder Rep. 53 (1995) 10-11.

[49] J.J. Fisher y R.M. Haeberle, Mod. Dev. Powder Metall. 21 (1988) 461-477.

[50] A. Bhadurl, V. Gopinathan, P. Ramakrishan y A.P. MIODOWNIK, Metall. Mater. Trans. A. 27 (1996) 37183726.

[51] D.G. KIM, J. Kaneko y M. Sugamata, Proc. PM'93, Kioto, Japón, 1993, Japan Society of Powder Metallurgy, Kioto, Japón, 1993, pp. 120-123.

[52] A. Bahduri, V. Gopinanathan, P. Ramakrishnan, G. Ede y A.P. MIODOWNIK, Scr. Metall. Mater. 28 (1993) 907912.

[53] E.M. Taleff, M. Nagao, Y. Ashida y O.D. Sherby, J. Mater. Res. 11 (1996) 2725-2730.

[54] C. Suryanarayana y F.H Froes, Adv. Mater. 5 (1993) 96. 
[55] C. Suryanarayana, R. Sundaresan y F.H. Froes, Mater. Sci. Eng. A. 150 (1992) 117.

[56] Z. Peng, C. Suryanarayana y F.H. Froes, Scr. Metall. Mater. 27 (1992) 475.

[57] S. Van Dyck, L. Delaey, L. Froyen y L. Buekenhout, Intermetallics. 3 (1995) 309.

[58] S. Van Dyck, I. Delaey, I. Froyen y I. Buekenhout, Proc. Euro PM'95, Birmingham, Reino Unido, 1995, EPMA, Shrewsbury, Reino Unido, 1995, pp. 517.

[59] C. Suryanarayana, G.H. Chen, A. Frefer y F.H. Froes, Mater. Sci. Eng. A. 158 (1992) 93.

[60] S.G. Pyo, H. Yasuda y D.T. Yamane, Mater. Sci. Eng. $179 / 180$ (1994) 676.

[61] A.K. Bhattacharya y E. Arzt, Scr. Metall. Mater. 127 (1992) 635.

[62] E. Hellstern y L. SChultz, J. Appl. Phys. 63 (1988) 1408.

[63] E. Hellstern y L. Schultz, Mater. Sci. Eng. 93 (1987) 213.

[64] C.E. Da Costa, J.M. Torralba, J.M. Ruiz-Prieto, J.M. Badía, V. Amigó, W.C. Zapata, Adv. Powder Metall. Particulate Mater. 1 (1996) 23-31.

[65] R.B. Bhagat, M.F. Amateau y E.C. Smith, Int. J. Powder Metall. 25 (1989) 311-316.
[66] D.O. Gotharg, Mod. Dev. Powder Metall. 21 (1988) 511-518.

[67] D.J. Chellman y T.D. Bayha, Adv. Powder Metall. Particulate Mater. 7 (1992) 257-269.

[68] L. LU, M.O. LAI Y S.H. YAP, Mater. Sci. Tech. 13 (1997) 192-202.

[69] A. LAWLEY Selected Case Studies in Powder Metallurgy. Ed. I. JENKINS y J.V. WOOD, The Institute of Metals, Londres, Reino Unido, 1991, pp. 66-75.

[70] P. Ramakrishnan, Adv. Powder Metall. Particulate Mater. 19 (1992) 237-249.

[71] A. Bhaduri, V. Gopinathan, P. Ramakrishan y A.P. MIODOWNIK, Adv. Powder Metall. Particulate Mater. 7 (1992) 285-301.

[72] K. TACHI Y K. KITA, Mater. Sci. Forum. 217-222 (1996) 1109-114.

[73] M.L. Van Meter, S.L. Kampe y A. Lawley, Adv. Powder Metall. Particulate Mater. 7 (1992) 285-301.

[74] P.G. MCCORMICK y F.H. FroEs, JOM 50 (1998) 61-65.

[75]، H.R. LASt y R.K. Garret, Metall. Mater. Trans. A. 27 (1996) 737-745. 